\title{
How are muscle synergies affected by electromyography pre-processing?
}

\author{
Paulina Kieliba, Peppino Tropea, Elvira Pirondini, Martina Coscia, Silvestro Micera, Fiorenzo Artoni
}

\begin{abstract}
Muscle synergies have been used for decades to explain a variety of motor behaviors, both in humans and animals and, more recently, to steer rehabilitation strategies. However, many sources of variability such as factorization algorithms, criteria for dimensionality reduction and data preprocessing constitute a major obstacle to the successful comparison of the results obtained by different research groups. Starting from the canonical EMG processing we determined how variations in filter cut-off frequencies and normalization methods, commonly found in literature, affect synergy weights and inter-subject similarity (ISS) using experimental data related to a 15-muscles upper-limb reaching task. Synergy weights were not significantly altered by either normalization (maximum voluntary contraction - MVC - or maximum amplitude of the signal - SELF) or band-pass filter $([20-500 \mathrm{~Hz}]$ or $[50-500]$ Hz). Normalization did however alter the amount of variance explained by a set of synergies, which is a criterion often used for model order selection. Comparing different low-pass (LP) filters $(0.5 \mathrm{~Hz}, 4 \mathrm{~Hz}, 10 \mathrm{~Hz}, 20 \mathrm{~Hz}$ cut-offs) we showed that increasing the low pass filter cut-off had the effect of decreasing the variance accounted for by a set number of synergies and affected individual muscle contributions. Extreme smoothing (i.e., LP cut-off $0.5 \mathrm{~Hz}$ ) enhanced the contrast between active and inactive muscles but had an unpredictable effect on the ISS. The results presented here constitute a further step towards a thoughtful EMG pre-processing for the extraction of muscle synergies.
\end{abstract}

Index Terms-Muscle Synergies, Upper limb, Arm-reaching movements, EMG, Factor Analysis, Data Pre-processing.

This work was supported by the Wyss Center for Bio and Neuroengineering and RONDA Regione Toscana, Bando FAS Salute 2014 PAR FAS 2007-2013. Dr. Artoni's contributions were also supported by the Marie Curie Individual Fellowship Grant H2020-MSCA-IF-2016 BIREHAB (Increasing the robustness and neural integration of BIdirectional prostheses for REHABilitation with robust and real time Independent Component Analysis), GA number 750947

P. Kieliba is with the Biomedical Signals and Systems, MIRA Institute for Biomedical Technology and Technical Medicine, University of Twente, Enschede, The Netherlands

P. Tropea is with The BioRobotics Institute, Scuola Superiore Sant'Anna, Pisa, Italy and the Department of Neurorehabilitation Sciences, Casa Cura Policlinico, Milano, Italy.

F. Artoni, E. Pirondini, M. Coscia and S.Micera are with Bertarelli Foundation Chair in Translational Neuroengineering, Center for Neuroprosthetics and Institute of Bioengineering, School of Engineering, École Polytechnique Fédérale de Lausanne (EPFL), Lausanne, Switzerland

M. Coscia is also with the Wyss Center for Bio- and Neuroengineering, Geneva, Switzerland

E. Pirondini is also with the Department of Radiology and Medical Informatics, University of Geneva, Geneva, Switzerland

F. Artoni and S. Micera are also with The BioRobotics Institute, Scuola Superiore Sant'Anna, Pisa, Italy

(correspondence to: fiorenzo.artoni@epfl.ch).

\section{INTRODUCTION}

$\mathrm{O}_{\mathrm{c}}^{\mathrm{N}} \mathrm{a}$ $\mathrm{NE}$ of the fundamental questions in motor control concerns the mechanisms underlying muscle coordination during the execution of movements [1]. The human musculoskeletal system is characterized by great redundancy, with more muscles than kinematic degrees of freedom [1-3], which makes it computationally complex to control each muscle independently. In 1967, Bernstein proposed the idea of modular organization of the muscle activity. He hypothesized that the control of movements may be based on a small set of muscular synergies combined in a task-dependent fashion to perform a desired action [4]. Muscle synergies can be defined as a group of muscles activated simultaneously with fixed relative gains. Each muscle synergy is composed of contributions from multiple muscles, with each muscle contributing to a different extent.

In general, identifying muscle synergies from raw electromyography (EMG) data involves following preprocessing steps: band-pass filtering, rectification, low-pass filtering, normalization, selection of the number of synergies to extract, and finally extraction of the synergies using a factorization algorithm of choice.

Linear factorization methods work by decomposing a set of pre-processed EMG signals and expressing them as the linear combination of basic activation coefficients and corresponding weight coefficients according to the equation

$$
M=W \cdot C+R E S,
$$

where $\mathrm{M}$ is the matrix $m x t$ of pre-processed EMG signals ( $m$ muscles recorded along a $t$-long time window); $\mathrm{C}$ is the $n x t$ matrix of the $n$ (with $n \leq m$ ) basic temporal components, also called activation coefficients; $\mathrm{W}$ is the $m x n$ matrix of the weight coefficients representing the algebraic transformation between the temporal components and the EMG signals; it highlights which muscles are working together and being functionally co-activated by a specific temporal component; RES is a residual term that accounts for noise. 
The muscle synergies concept has been widely used both to explain a variety of motor behaviors (e.g., reaching [5-8], posture [9], locomotion [10-13]) and animals (e.g., primate grasping [14] frog jumping [15, 16], cats' locomotion [17]), to study pathologies (e.g., to determine age-related [18, 19] or pathology-related modifications in muscles co-activations [20] and to predict the degree of impairment of stroke patients [2125]). Muscle synergies (and factorization methods in general) can also be viewed as a useful tool to reveal underlying patterns in muscle activity that may reflect different levels of neural functions [26, 27], to compare performance across subjects [28] and to reduce the input features for a classifier $[29,30]$.

All these studies support the hypotheses of a neural control of movements based on the muscle synergies and the possibility to extract them from recordings of superficial muscle activity. However, it is often difficult to successfully compare the results obtained by different research groups due to the variability in the EMG processing methods (e.g. the choice of filtering cut-off frequencies, normalization strategies, rectification), factorization algorithms for synergies extraction (e.g., Nonnegative Matrix Factorization (NMF) [31, 32], Principal Component Analysis (PCA) [32-35], Independent Component Analysis (ICA) [36-39], Factor Analysis (FA) [40]) and different criteria for selecting the number of synergies to extract (e.g., Explained Variance [5, 21], Minimization of Variance Accounted For (VAF) [41], Eigenvalue Criterion [19, 42, 43], Cluster Analysis [44]).

Tresch et al. [32] identified a few best-performing factorization algorithms that yielded similar muscle synergies, suggesting that muscle synergies reflect indeed basic features of the organization of muscle activation and can be extracted by blind source separation (BSS) methods. However, the choice of different EMG pre-processing techniques, by altering the data information content available to the factorization algorithm, might alter its performance, the number of synergies to extract and the weights and activation coefficients of the synergies themselves [45]. For instance, Hug et al. [46], Krogt et al.[47], Shuman et al. [48], recently showed that smoothing influences the number of extracted muscle synergies (i.e., the VAF by a given number of synergies decreased when increasing the LP cut-off frequency). Artoni et al. [44] showed how a different number of extracted synergies could lead to different interpretation of the results. In other words, depending on the researcher's selection of pre-processing steps, different conclusions and clinical interpretations might be drawn from the data [44].

Given the importance of these aspects, there is an increasing need to test the robustness of results to changes in the preprocessing pipeline, as suggested in [44, 47-49]. We hypothesize that each pre-processing step may have an effect on the number of synergies as well as on their structure, thus influencing the interpretation of the results.

For this reason, in the present study we have assessed the influence of various pre-processing methods on the muscle synergies extraction. This analysis was performed on experimental datasets from healthy subjects during point-to-

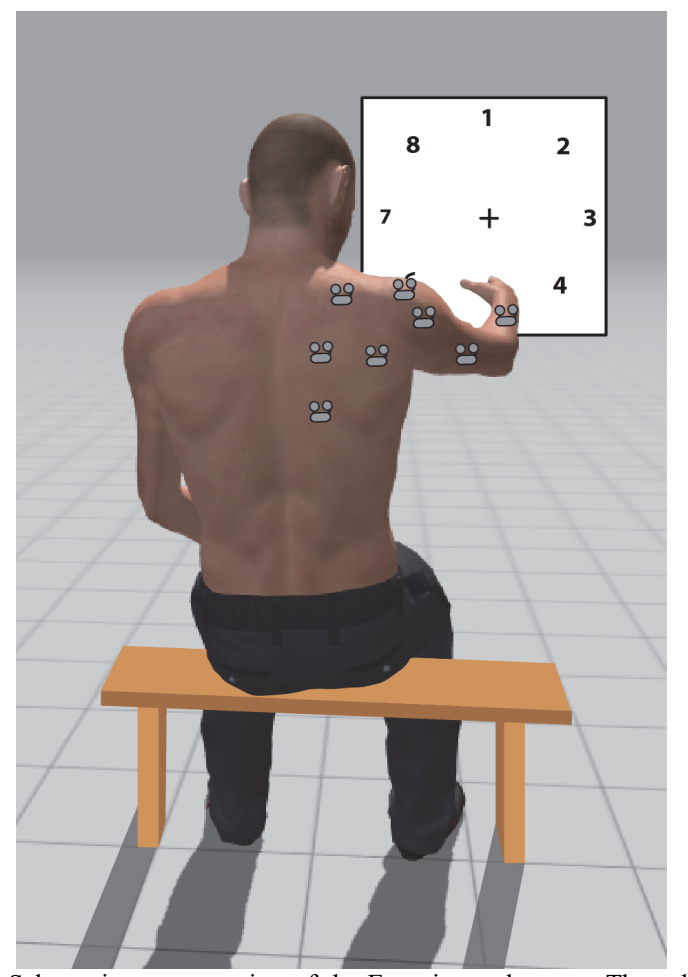

Fig 1. Schematic representation of the Experimental set up. The subject is seated in front of a target panel positioned at arm's length, centered and aligned with the subject's right shoulder acromion. The panel has eight targets arranged in a clock-like fashion. Wireless EMG bipolar sensors are placed on selected muscles. The picture shows only a few sensors placed on arbitrary muscles for demonstration purposes. See Table I for the full list of recorded muscles.

point 3D reaching movements. The results might help in comparing existing studies and can thus lead to a more consistent application of the muscle synergies methodology.

\section{MATERIALS AND METHODS}

\section{A. Participants}

Eight right-handed healthy male young subjects (age: $24.42 \pm 1.81$ years; height: $177.42 \pm 7.80 \mathrm{~cm}$; weight: $72.07 \pm 6.35 \mathrm{~kg}$ ) were enrolled in the study. They did not present any evidence of skeletal, neurological or vascular diseases, and exhibited normal range of motion and muscle strength. The study was carried out in the Translational Neural Engineering Laboratory at the Ecole Polytechnique Federale de Lausanne (EPFL) after approval by the EPFL BMI Ethics Committee for Human Behavioral Research. The recordings were carried out in agreement with the Declaration of Helsinki. All participants signed an informed consent before starting the experimental sessions.

\section{B. Experimental protocol}

The experimental set-up and the protocol were the same as those used in our previous studies $[38,50,51]$ and similar to the setups used in Cheung et al. [21], and Coscia et al [52]. The subject sat in front of a target panel. The center of the target panel was aligned with his right shoulder acromion. The panel had eight targets arranged in a clock-like fashion and was positioned at a distance of $20 \mathrm{~cm}$ from its center. The 
distance between the subject and the panel was equal to the participant's arm length. The starting position was located mid-way from the center of the target panel and the subject's acromion. The subject was instructed to reach, in a randomized order and at a self-chosen pace, to each of the eight targets on the target panel. The reaching movements were performed from the starting position toward the selected target and then back to the starting position. Each reaching movement (starting position - target - starting position) constituted a trial. The task was repeated 12 times for a total of 96 trials (Fig 1).

During those trials, the EMG activity was acquired from 15 upper limb muscles (see Table I) using superficial $\mathrm{Ag}-\mathrm{AgCl}$ electrodes (Kendall H124SG, ECG electrodes) with an interelectrode spacing of $2 \mathrm{~cm}$. The skin was cleaned and shaved, and the electrodes were placed, whenever possible, according to the standard procedure for Surface Electromyography for Non-Invasive Assessment of Muscles (SENIAM) guidelines [53]. The EMG signals were acquired at $3000 \mathrm{~Hz}$ with a wireless Noraxon Desktop DTS system (Scottsdale, Arizona) and an on-board $1^{\text {st }}$ order $10 \mathrm{~Hz}$ high-pass filter.

Before starting the task, the subjects were asked to perform an isometric maximum voluntary contraction (MVC) test. Since in our study only healthy subjects were recruited, it was feasible to obtain the MVC for all the included muscles. We followed the procedure described in the SENIAM guidelines [53] for the triceps brachii, the biceps brachii short head, the biceps brachii long head, the superior trapezius, the deltoid anterior, medial, and posterior. As for the other muscles, we used the standard procedures described by Clarkson et al. and Daniels et al. $[54,55]$. The tests were executed by the same experimenter for all the subjects. We repeated each test three times, with a break between each contraction to prevent muscle fatigue. For each muscle the MVC was computed as the maximum value obtained from the MVC data, preprocessed in the same way as the task EMG data [52].

\section{Pre-processing}

The EMG pre-processing was performed by using custom routines written in Matlab (Mathworks Inc., Natick, MA, USA). For each subject, the EMG data from all 12 trials were first concatenated. According to the current state-of-the-art [1, $32,56]$, muscle synergies were derived separately for each subject from concatenated EMG signals that were preprocessed in the following way:

1. Band-pass (BP) filtering (zero-phase $6^{\text {th }}$ order, Butterworth [57]): it removes drifts, aliasing effects, high-frequency noise (low-pass) and, to some extent, movement artifacts (high-pass).

2. Rectification: it is a commonly-used non-linear preprocessing step that allows to compute the EMG envelope. It is deemed useful to enhance the firing rate information of the signal by shifting the peak of the Motor Unit Action Potential spectrum toward the lower firing rate frequencies, whilst maintaining the firing rate spectra [58].

3. Low-pass (LP) filtering (zero-phase $4^{\text {th }}$ order,
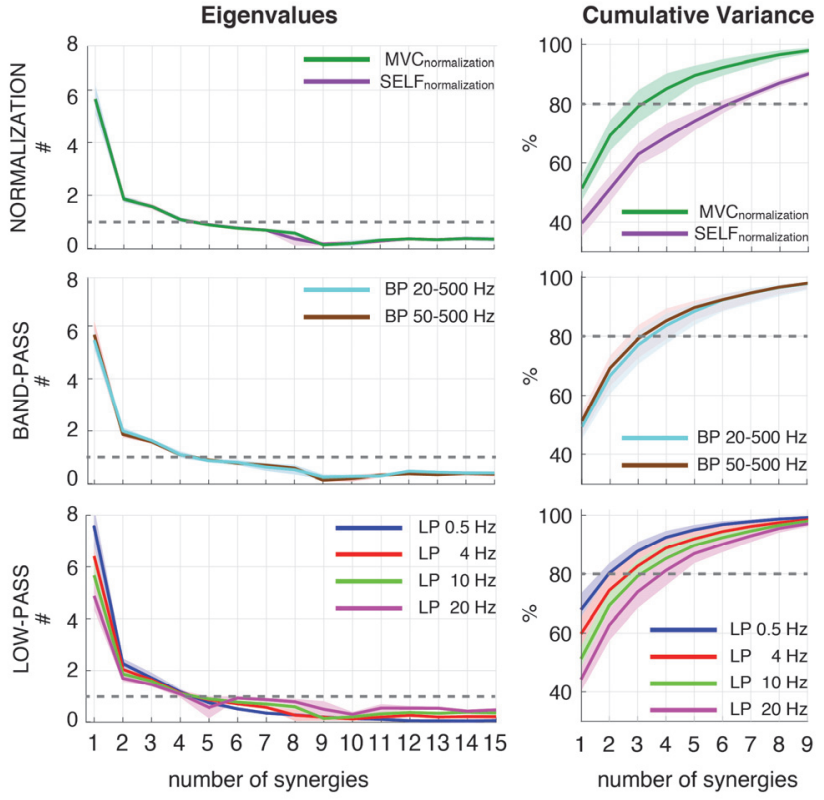

Fig 2. Eigenvalues and cumulative variance for each pre-processing condition. Median values across subjects (thick lines) and median absolute deviation (shaded areas) of the eigenvalues (left column) and cumulative variance (right column) as a function of the number of extracted synergies in different normalizations (top), BP filters (middle) and LP filters (bottom) conditions. The threshold for determining the number of synergies to extract, according to each criterion (respectively Eigenvalue $\geq 1$, and Cumulative Explained Variance $>80 \%$ ) are indicated by the dotted line.

Butterworth): applied to the rectified EMG signal, it ensures that no high frequency content alters the envelope shape. The lower the cut-off the smoother the envelope.

4. Normalization: by the Maximum Voluntary Contraction (MVC) or by maximum amplitude of the signal itself (SELF) during the task. It assigns equal initial importance to each muscle, i.e., allowing for non-biased synergy extraction.

\section{Muscle synergies extraction}

Muscle synergies extraction comprised the following steps:

5. Selection of the number of components (synergies) to retain: it is performed to avoid extracting noisy components (overfitting).

6. Synergies extraction via FA to disentangle information from noise and to identify co-contractions across muscles.

Muscle synergies were extracted here using Factor Analysis (FA) with "varimax" rotation. This algorithm has been shown to be very reliable for muscle synergies extraction [32] and to provide results equivalent to the commonly used NMF algorithm [23, 32, 59]. The number of extracted factors (muscles synergies i.e., the dimensionality of the muscle activity subspace identified by the algorithm) was chosen based on two alternative criteria:

i. the Eigenvalue $\geq 1$ criterion; it is based on the assumption that a factor can only be considered significant if its explained variance is equal or higher to that of the 


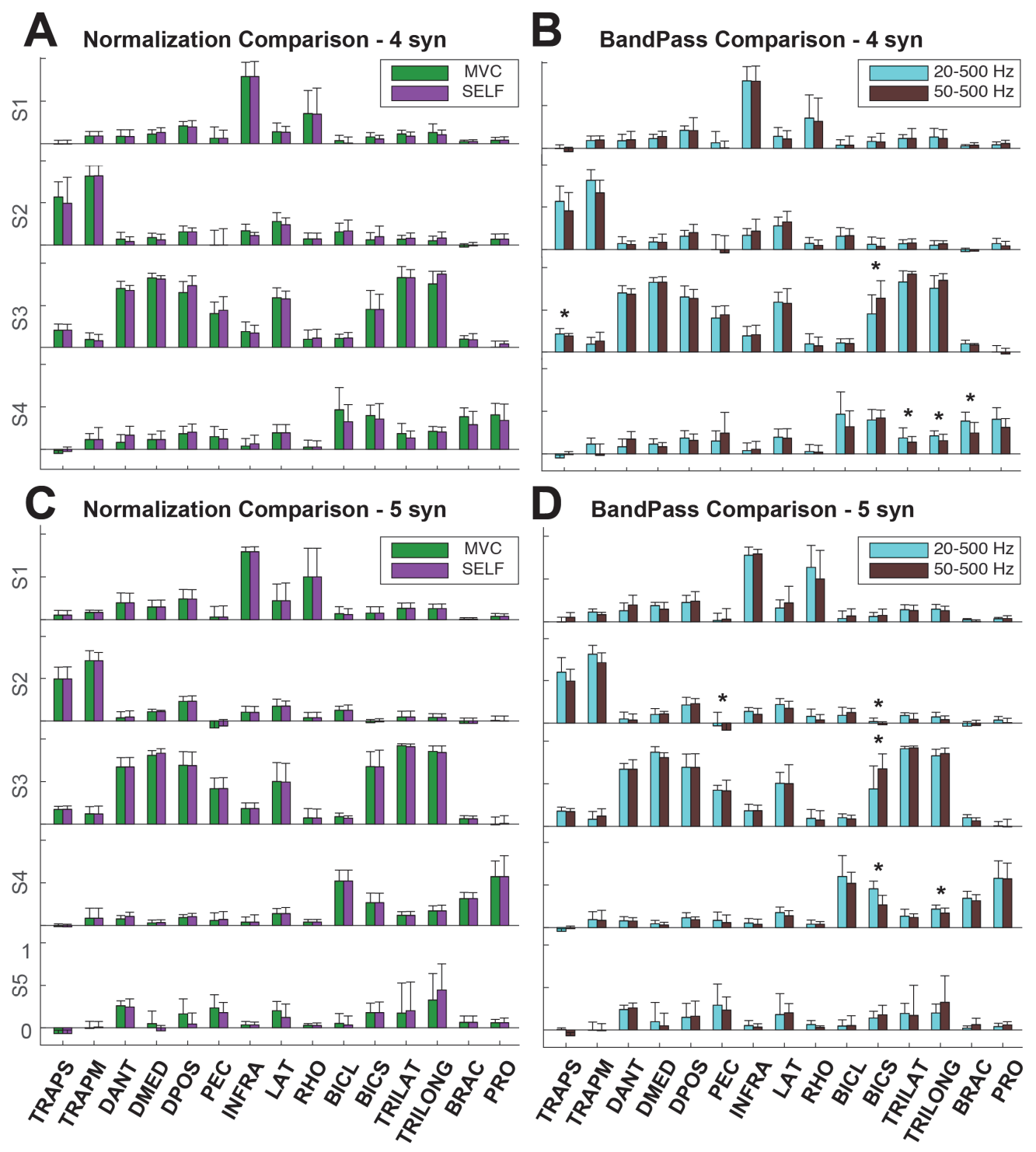

Fig 3. Effects of different normalization and BP filters conditions on the muscle synergies structure. Weight coefficients for four (first row) and five (second row) extracted muscle synergies (S1-S5). Panels A and C show the influence that two different normalization techniques of the EMG signal (i.e., to MVC and to SELF) hold on the weighting coefficients. Panels B and D illustrate the effect of the BP filters, (i.e., 20-500 Hz and 50-500 Hz) on the weighting coefficients. The bars show the group median and the lines indicate the median absolute deviations. Significant results (Wilcoxon paired signed rank test, $\mathrm{p}<0.05$ ) are marked with *.

original variable $[19,42,43]$;

ii. the cumulative explained variance (R2) criterion; it is based on the assumption that the number of synergies account for at least $80 \%$ of the variance (i.e., $\mathrm{R} 2 \geq 80 \%$ ) of the EMG envelope time [5, 52].

\section{E. Pre-processing tests}

In order to determine the effects of different pre-processing methods only one parameter at a time was changed with respect to the default parameters: $6^{\text {th }}$ order $[50-500] \mathrm{Hz}$ Butterworth BP filter; 4th order Butterworth LP filter with $10 \mathrm{~Hz}$ cut-off frequency, MVC data for normalization [21, 24]. The following tests were performed:

i. [50-500] Hz vs. [20-500] Hz BP filter in order to compare the default BP filter with a more conservative one. This comparison enables to find the compromise between a
TABLE I

\begin{tabular}{ll}
\multicolumn{2}{c}{ ACRONYMS OF THE MUSCLES RECORDED } \\
\hline Acronym & \multicolumn{1}{c}{ Muscle } \\
\hline DANT & Deltoid Anterior \\
DMED & Deltoid Medial \\
DPOS & Deltoid Posterior \\
PEC & Pectoralis Major \\
LAT & Latissimus Dorsi \\
INFRA & Infraspinatus \\
RHO & Rhomboid Major \\
PRO & Pronator Tensor \\
BICS & Biceps Brachii Short Head \\
BICL & Biceps Brachii Long Head \\
BRAC & Brachialis \\
TRILAT & Lateral Head of Triceps Brachii \\
TRILONG & Long Head of Triceps Brachii \\
TRAPS & Superior Trapezius \\
TRAPM & Medial Trapezius \\
\hline \hline
\end{tabular}


higher data stationarity (narrower band filter, such as [50 - 500] Hz) and greater amount of EMG information retained (wider band filter, such as [20 - 500] Hz).

ii. $10 \mathrm{~Hz}$, vs. $0.5 \mathrm{~Hz}, 4 \mathrm{~Hz}, 20 \mathrm{~Hz}$ LP filter cut-off frequency after rectification (without changing either the order or the type of the filter). This comparison enables to estimate the maximum acceptable smoothing that would not remove useful information from the data.

iii. MVC normalization vs. SELF normalization; here the standard MVC normalization is compared to the normalization performed according to the maximum value reached by each muscle during the task performance. This comparison enables the researchers to make informed decisions about which type of normalization is best suited for their study.

\section{F. Statistical Analysis for the assessment of the pre- processing effects}

For each number of synergies considered the effect of Normalization and BP filter on the number of synergies to be extracted using Eigenvalue and Cumulative Variance criteria were assessed using a Wilcoxon paired signed rank test as data were not distributed in a Gaussian fashion (Shapiro-Wilk test, significance $\alpha=0.05$ ). Similarly, the effect of different LP filters was determined via the Friedman test and the Spearman's rank correlation coefficient.

In order to compare the synergy weights across preprocessing conditions, for each number of retained synergies ( 4 or 5 - see results), we calculated the median and absolute median deviation (MAD) of the weights over all the subjects. The number of synergies was kept constant across subjects and conditions to simplify the analyses and allow for direct statistical comparison of weight coefficients as already proposed in other works [21, 23, 38, 52].

For each pre-processing condition the synergies were categorized across subjects into groups with a hierarchical clustering procedure based on the minimization of the Minkowski distance between vectors [21]. The number of clusters specified was set to be the same as the number of muscle synergies extracted, so that each cluster contained exactly one synergy per subject. The muscle synergies were matched across conditions by using the normalized scalar product (DOT).

The effect of different BP filters and normalization methods on the weight coefficients of extracted muscle synergies (across subjects) was assessed using a Wilcoxon paired signed rank test. This was done separately for each muscle and each synergy. Similarly, the Friedman test was used to compare the effect of different LP filters. When a significant effect was found, the Kendall's W coefficient was calculated to assess the effect size. We furthermore corroborated the statistical tests by computing the DOT between the homologous synergies (see Results).

The degree of similarity of the synergies across subjects (within each cluster) was defined as the median value of the scalar products calculated between weight coefficients normalized to the Euclidean norm of each two homologous muscle synergies in the given cluster (mDOT). The effects of Normalization and BP filter on the inter-subject similarity (ISS) were assessed using a Wilcoxon paired signed rank test Similarly, the effect of different LP filters on the ISS was assessed using the Friedman test. When a significant effect was found, the Kendall's W coefficient was calculated to assess the effect size. Post-hoc analysis was carried out with Bonferroni correction for multiple comparisons (6 comparisons resulting in the significance level $\alpha=0.0083$ ).

Finally, the effect of the number of synergies extracted (4 synergies vs 5 synergies) on the ISS was assessed using a Wilcoxon paired signed rank test separately for each of the synergies. Here, the effect size was calculated as $r=Z$-stat $/ \sqrt{ } N$.

All the statistical tests were performed with SPSS (Statistical Package for Social Science, IBM, Armonk, New York, USA) with significance level set at $\alpha=0.05$.

\section{RESULTS}

\section{A. Muscle synergies structure}

The extracted muscle synergies (Figures 3 and 4) exhibit the following characteristics:

i. S1 mainly involves INFRA and RHO muscles (the latter with a great variability across subjects). This synergy contributes to performing abduction and external rotation of the upper arm.

ii. S2 reflects the involvement of the trapezius muscles (i.e., TRAPS and TRAPM) in the elevation of the arm.

iii. S3 is mainly characterized by the activity of the muscles deputed to the flexion-extension of arm (both head of triceps TRILAT and TRILONG and BICS), to the flexion of the shoulder (DMED and with a smaller degree DANT and DPOS), and to the stabilization and the postural support of the arm (PEC and LAT). This synergy facilitates forward arm elevation and elbow flexion and extension.

iv. S4 mainly involves BICL and PRO and, though to a lesser extent, BRA and the other head of biceps (BICS). It can be ascribed to the coordination of elbow flexors while executing point-to-point reaching movements.

v. S5 accounts mostly for the contribution of TRILAT and TRILONG to the extension of the elbow. It is, however, characterized by great variability across subjects.

\section{B. Effects of pre-processing on estimation of the number of muscle synergies}

The different BP, LP filters and normalization methods did not have significant influence on the number of synergies to be extracted according to the Eigenvalue criterion (Fig 2, Panel A), which was also less affected by variability across subjects with respect to the cumulative variance criterion. Conversely, the cumulative variance criterion was found to be strongly affected by the normalization type $(Z=2.555, \mathrm{p}=$ $0.011)$. On average, three more synergies were needed to explain the same amount of variance when SELF normalization was employed (Fig 2, Panel B). Similarly, LP 


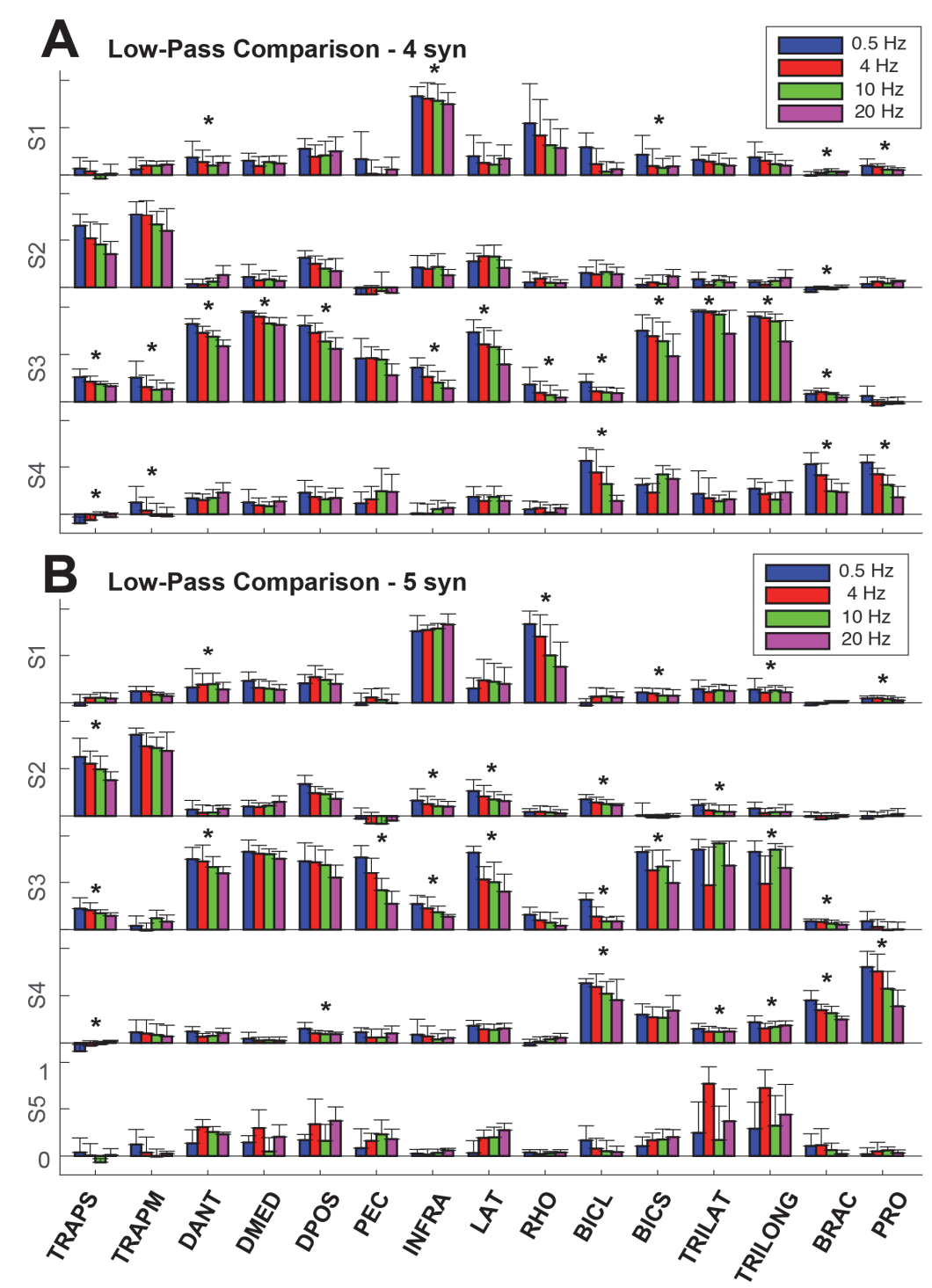

Fig 4. The effect of different LP filters on the muscle synergies. Weight coefficients for (A) four and (B) five extracted muscle synergies (S1-S5). Panels illustrate the effect of the different LP filters, (i.e., $0.5,4,10,20 \mathrm{~Hz}$ ) on the muscle synergies. The bars show the group medians, the lines the median absolute deviations. Data are shown for the same muscles (labels on the horizontal axis) of Figure 3 . Significant results (Friedman test, $\mathrm{p}<0.05$ ) are marked with *.

filtering cut-off frequency was found to be inversely correlated to the amount of variance that a set of synergies can explain (Spearman $\operatorname{rank} r_{s}(58)=0.2892, p=0.025$ ), thus significantly affecting the number of synergies to be extracted $(\chi(3)=21.986, p=66 \mathrm{e}-05)$.

The choice of four and five synergies respectively fulfilled the Eigenvalue criterion and Cumulative Explained Variance criterion for all the pre-processing conditions. Therefore, both four and five synergies were considered in this study.

The structure of the first four synergies (S1-4 Fig 3, panels A-B) was not modified by the extraction of a further fifth one (S5 Fig 3, panels C-D).

\section{Effects of pre-processing on muscle synergy weights}

As revealed by the Wilcoxon paired signed rank test and by the DOT products neither normalization (median DOT across pre-processing conditions equal to 1) nor BP filtering (median DOT $>0.95$ ) had any significant effect on synergies weights
(Fig 3). Although some differences could be ascribed to BP cut-off frequency, they were either isolated (BICS in S3), referred to low weight muscles (TRILAT, TRILONG, BRAC in S4) or were not robust to changes in the number of synergies extracted (PEC, BICS in S2-Panel B vs. S2-Panel A) and they are, thus, likely due to the stochastic nature of the EMG [43].

Conversely, LP filtering had a significant effect on synergies weights, both when extracting four and five synergies (Fig 4). An increase in LP filtering cut-off frequency reduced the smoothness of the EMG envelope and significantly $(p<0.05)$ decreased the relative weights of the majority of the most active muscles. Kendall's W calculated for the significantly affected weights ranged from 0.381 to 0.956 (mean: 0.567; standard deviation: 0.188), indicating moderate to strong effect sizes. Those significant differences were particularly noticeable e.g., for DANT, DMED, DPOS and LAT of synergy S3, BRAC and PRO for synergy S4. This 
TABLE II

THE EFFECT OF DIFFERENT LP FILTERS ON INTER-SUBJECT SIMILARITY

4 SYNERGIES

\begin{tabular}{|c|c|c|c|c|c|c|c|c|c|c|c|c|}
\hline & LP $0.5 \mathrm{~Hz}$ & LP $4 \mathrm{~Hz}$ & LP $10 \mathrm{~Hz}$ & LP $20 \mathrm{~Hz}$ & p-value & $\begin{array}{c}\text { effect } \\
\text { size }\end{array}$ & $\begin{array}{l}\text { Norm to } \\
\text { MCV }\end{array}$ & $\begin{array}{l}\text { Norm to } \\
\text { MAX }\end{array}$ & $\begin{array}{c}\text { p- } \\
\text { value }\end{array}$ & $\begin{array}{c}\text { BP } \\
20-500 \mathrm{~Hz}\end{array}$ & $\begin{array}{c}\text { BP } \\
50-500 \mathrm{~Hz}\end{array}$ & $\begin{array}{c}\mathrm{p}- \\
\text { value }\end{array}$ \\
\hline S1 & $0.511 \pm 0.27$ & $0.397 \pm 0.35$ & $0.437 \pm 0.32$ & $0.560 \pm 0.26$ & 0.055 & $\mathrm{n} / \mathrm{a}$ & $0.456 \pm 0.24$ & $0.324 \pm 0.17$ & 0.48 & $0.437 \pm 0.32$ & $0.456 \pm 0.24$ & 0.29 \\
\hline $\mathrm{S} 2$ & $0.696 \pm 0.16$ & $0.731 \pm 0.15$ & $0.796 \pm 0.09$ & $0.560 \pm 0.35$ & 0.150 & $\mathrm{n} / \mathrm{a}$ & $0.800 \pm 0.13$ & $0.206 \pm 0.53$ & 0.86 & $0.796 \pm 0.10$ & $0.800 \pm 0.13$ & 0.68 \\
\hline S3 & $0.903 \pm 0.03$ & $0.883 \pm 0.05$ & $0.892 \pm 0.09$ & $0.863 \pm 0.05$ & 0.142 & $\mathrm{n} / \mathrm{a}$ & $0.890 \pm 0.04$ & $0.888 \pm 0.05$ & 0.33 & $0.892 \pm 0.05$ & $0.889 \pm 0.04$ & 0.17 \\
\hline S4 & $0.680 \pm 0.13$ & $0.546 \pm 0.18$ & $0.539 \pm 0.19$ & $0.478 \pm 0.11$ & $0.001 * *$ & $\begin{array}{c}0.19 \\
\text { (small) }\end{array}$ & $0.579 \pm 0.16$ & $0.533 \pm 0.12$ & 0.19 & $0.539 \pm 0.14$ & $0.579 \pm 0.16$ & $0.01 *$ \\
\hline
\end{tabular}

5 SYNERGIES

\begin{tabular}{|c|c|c|c|c|c|c|c|c|c|c|c|c|}
\hline & LP $0.5 \mathrm{~Hz}$ & LP $4 \mathrm{~Hz}$ & LP $10 \mathrm{~Hz}$ & LP $20 \mathrm{~Hz}$ & p-value & $\begin{array}{c}\text { effect } \\
\text { size }\end{array}$ & $\begin{array}{c}\text { Norm to } \\
\mathrm{MCV}\end{array}$ & $\begin{array}{c}\text { Norm to } \\
\text { MAX }\end{array}$ & $\begin{array}{c}\mathrm{p}- \\
\text { value }\end{array}$ & $\begin{array}{c}\text { BP } \\
20-500 \mathrm{~Hz}\end{array}$ & $\begin{array}{c}\mathrm{BP} \\
50-500 \mathrm{~Hz} \\
\end{array}$ & $\begin{array}{c}\mathrm{p}- \\
\text { value }\end{array}$ \\
\hline S1 & $0.612 \pm 0.24$ & & $0.736 \pm 0.16$ & $0.671 \pm 0.20$ & 0.372 & $\mathrm{n} / \mathrm{a}$ & $0.736 \pm 0.16$ & $0.579 \pm 0.16$ & 0.27 & $0.545 \pm 0.29$ & $0.736 \pm 0.16$ & $0.01 *$ \\
\hline S2 & $0.717 \pm 0.13$ & $0.806 \pm 0.09$ & $0.832 \pm 0.05$ & $0.798 \pm 0.11$ & $0.002 * *$ & $\begin{array}{c}0.175 \\
\text { (small) }\end{array}$ & $0.832 \pm 0.05$ & $0.833 \pm 0.05$ & 0.89 & $0.768 \pm 0.15$ & $0.832 \pm 0.05$ & $0.01 *$ \\
\hline S3 & $0.907 \pm 0.05$ & $0.844 \pm 0.13$ & $0.859 \pm 0.05$ & $0.856 \pm 0.08$ & $0.009 * *$ & $\begin{array}{c}0.160 \\
\text { (small) }\end{array}$ & $0.859 \pm 0.05$ & $0.857 \pm 0.05$ & 0.06 & $0.900 \pm 0.04$ & $0.859 \pm 0.05$ & 0.12 \\
\hline S4 & $0.793 \pm 0.05$ & $0.837 \pm 0.05$ & $0.814 \pm 0.05$ & $0.658 \pm 0.18$ & $0.018^{*}$ & $\begin{array}{c}0.120 \\
\text { (small) }\end{array}$ & $0.814 \pm 0.05$ & $0.785 \pm 0.05$ & $0.01 *$ & $0.765 \pm 0.10$ & $0.814 \pm 0.05$ & 0.78 \\
\hline S5 & $0.162 \pm 0.28$ & $0.208 \pm 0.46$ & $0.375 \pm 0.21$ & $0.461 \pm 0.15$ & $0.006^{* *}$ & $\begin{array}{c}0.147 \\
\text { (small) } \\
\end{array}$ & $0.375 \pm 0.05$ & $0.369 \pm 0.05$ & 1 & $0.199 \pm 0.28$ & $0.375 \pm 0.05$ & 0.54 \\
\hline
\end{tabular}

Median dots and absolute median deviations respectively for four (top) and five (bottom) extracted muscle synergies (S1-S5, rows) obtained with different LP cut-off frequencies, normalization methods and BP cut-off frequencies (columns). The last columns in each category show the p-values (Friedman test/Wilcoxon signed rank test) with significant comparisons $(\mathrm{p}<0.05)$ are marked by *, and associated effect sizes $($ Kendall's $\mathrm{W})$.

was also reflected in the DOTs values (see Table III). For example DOT $(0.5 \mathrm{~Hz}-20 \mathrm{~Hz})<$ DOT $(4 \mathrm{~Hz}-20 \mathrm{~Hz})<$ DOT $(10 \mathrm{~Hz}-20 \mathrm{~Hz})$ in every instance.

\section{Inter-subject similarity}

As for the inter-subject similarity, when 4 synergies were extracted only the similarity of the weights of S4 was significantly influenced by the choice of the LP filter ( $p$-values and associated effect sizes reported in Table II). With 5 synergies S2, S3, S4, S5 were all affected. Both for S4 (four synergies) and S2, S3, S4, S5 (five synergies), post-hoc analysis (with Bonferroni correction for multiple comparisons - significance $\alpha=0.0083$ ), showed significant effects of 0.5 $\mathrm{Hz}$ LP filter on the inter-subject similarity with respect to other conditions: in case of 5 synergies lower ISS for S2 and S5, higher ISS for S3 and S4; in case of 4 synergies higher ISS for S4 (Table 2). Table 2 shows that SELF normalization slightly decreased inter-subject similarity but this result reached significance only for S4 with 5 extracted synergies. Higher cut-off of the BP filter $[50-500 \mathrm{~Hz}]$ significantly increased inter-subject similarity in three instances (S4 - four synergies extracted - S1, S2 - five synergies extracted)

As for the number of synergies, although when five synergies are extracted the effect of LP cutoff is more prominent, overall when only four synergies were extracted the inter-subject similarity in the weight coefficients was significantly lower $(Z<87, p<0.008)$ than with five synergies for $\mathrm{S} 1, \mathrm{~S} 2$ and $\mathrm{S} 4(\mathrm{r}>0.559$, indicating strong effect size).

\section{Discussion}

In this study we investigated how different pre-processing of the EMG signals affects the muscle synergies' structure and number, as well as their variability across subjects. We have also evaluated the pre-processing's influence on two criteria for determining the number of synergies (i.e., Eigenvalues and Cumulative Variance). The synergies extracted in this study are stable across participants and conditions. They are physiologically plausible for explaining the motor activation during the reaching movements and similar in dimensionality and structure to those found in the literature $[5,6,21,22,25$, 52]. We used FA for the extraction of the muscle synergies. FA is a multivariate statistical technique able to identify latent variables underlying the linear relationships existing among a large set of correlated variables [43]. FA has been used to study human locomotion, reaching/grasping, cycling, posture and neuro-rehabilitation [12, 13, 25, 40, 42, 43, 60-64]. While the implementation of FA and NMF, as well as the statistical principles on which they are based are different, both FA and NMF have been shown to produce equivalent results on both simulated [32] and real data [59].

Regarding LP cut-off frequencies, we chose the values best represented in the literature, i.e., $4 \mathrm{~Hz}[24], 10 \mathrm{~Hz}[65,66], 20$ $\mathrm{Hz}[19,21]$. Similarly, for BP both lower $(20 \mathrm{~Hz})[59,67,68]$ and higher $(40 \mathrm{~Hz})[24,69]$ high-pass cut-offs have been used. Furthermore, a value of $0.5 \mathrm{~Hz}$ was chosen to clearly mark the effect of an extreme smoothing on the extracted muscle synergies

\section{A. LP filtering alters synergy weights and explained variance}

The most important result we found is that the LP filter does not only impact on the dimensionality of the data [46] but it also significantly influences the weights of the extracted synergies. The higher the cut-off of the LP filter the lower the weights of the dominant muscles contributing to the synergies (Fig 4), as well as the cumulative variance explained by a set number of extracted synergies. In this sense, higher LP cut- 
TABLE III

DOTS BETWEEN SYNERGIES WITH DIFFERENT LP CUT-OFF FREQUENCIES

\begin{tabular}{|c|c|c|c|c|c|c|c|c|c|c|}
\hline & \multicolumn{5}{|c|}{4 SYNERGIES } & & \multicolumn{4}{|c|}{5 SYNERGIES } \\
\hline & & $\begin{array}{c}\text { LP } \\
0.5 \mathrm{~Hz}\end{array}$ & $\begin{array}{c}\mathrm{LP} \\
4 \mathrm{~Hz} \\
\end{array}$ & $\begin{array}{c}\text { LP } \\
10 \mathrm{~Hz}\end{array}$ & $\begin{array}{c}\text { LP } \\
20 \mathrm{~Hz}\end{array}$ & & $\begin{array}{c}\text { LP } \\
0.5 \mathrm{~Hz}\end{array}$ & $\begin{array}{c}\mathrm{LP} \\
4 \mathrm{~Hz} \\
\end{array}$ & $\begin{array}{c}\text { LP } \\
10 \mathrm{~Hz}\end{array}$ & $\begin{array}{c}\text { LP } \\
20 \mathrm{~Hz}\end{array}$ \\
\hline \multirow{4}{*}{ S1 } & LP $0.5 \mathrm{~Hz}$ & 1 & $0.99 \pm 0.00$ & $0.91 \pm 0.08$ & $0.95 \pm 0.03$ & LP $0.5 \mathrm{~Hz}$ & 1 & $0.99 \pm 0.01$ & $0.92 \pm 0.06$ & $0.89 \pm 0.09$ \\
\hline & LP $4 \mathrm{~Hz}$ & & 1 & $0.98 \pm 0.02$ & $0.98 \pm 0.01$ & LP $4 \mathrm{~Hz}$ & & 1 & $1.00 \pm 0.00$ & $0.99 \pm 0.01$ \\
\hline & LP $10 \mathrm{~Hz}$ & & & 1 & $1.00 \pm 0.00$ & LP $10 \mathrm{~Hz}$ & & & 1 & $1.00 \pm 0.00$ \\
\hline & LP $20 \mathrm{~Hz}$ & & & & 1 & LP $20 \mathrm{~Hz}$ & & & & 1 \\
\hline \multirow{4}{*}{ S2 } & LP $0.5 \mathrm{~Hz}$ & 1 & $0.98 \pm 0.01$ & $0.96 \pm 0.02$ & $0.91 \pm 0.06$ & LP $0.5 \mathrm{~Hz}$ & 1 & $0.98 \pm 0.02$ & $0.97 \pm 0.02$ & $0.92 \pm 0.02$ \\
\hline & LP $4 \mathrm{~Hz}$ & & 1 & $0.99 \pm 0.00$ & $0.97 \pm 0.02$ & LP $4 \mathrm{~Hz}$ & & 1 & $1.00 \pm 0.00$ & $0.95 \pm 0.03$ \\
\hline & LP $10 \mathrm{~Hz}$ & & & 1 & $0.99 \pm 0.01$ & LP $10 \mathrm{~Hz}$ & & & 1 & $0.96 \pm 0.01$ \\
\hline & LP $20 \mathrm{~Hz}$ & & & & 1 & LP $20 \mathrm{~Hz}$ & & & & 1 \\
\hline \multirow{4}{*}{ S3 } & LP $0.5 \mathrm{~Hz}$ & 1 & $0.99 \pm 0.00$ & $0.99 \pm 0.00$ & $0.97 \pm 0.01$ & LP $0.5 \mathrm{~Hz}$ & 1 & $0.98 \pm 0.01$ & $0.99 \pm 0.00$ & $0.95 \pm 0.04$ \\
\hline & LP $4 \mathrm{~Hz}$ & & 1 & $1.00 \pm 0.00$ & $0.99 \pm 0.01$ & LP $4 \mathrm{~Hz}$ & & 1 & $1.00 \pm 0.00$ & $0.97 \pm 0.03$ \\
\hline & LP $10 \mathrm{~Hz}$ & & & 1 & $0.99 \pm 0.00$ & LP $10 \mathrm{~Hz}$ & & & 1 & $0.99 \pm 0.01$ \\
\hline & LP $20 \mathrm{~Hz}$ & & & & 1 & LP $20 \mathrm{~Hz}$ & & & & 1 \\
\hline \multirow{4}{*}{ S4 } & LP $0.5 \mathrm{~Hz}$ & 1 & $0.98 \pm 0.01$ & $0.97 \pm 0.02$ & $0.91 \pm 0.05$ & LP $0.5 \mathrm{~Hz}$ & 1 & $0.99 \pm 0.00$ & $0.97 \pm 0.02$ & $0.94 \pm 0.04$ \\
\hline & LP $4 \mathrm{~Hz}$ & & 1 & $0.99 \pm 0.01$ & $0.96 \pm 0.03$ & LP $4 \mathrm{~Hz}$ & & 1 & $1.00 \pm 0.00$ & $0.98 \pm 0.01$ \\
\hline & LP $10 \mathrm{~Hz}$ & & & 1 & $0.98 \pm 0.02$ & LP $10 \mathrm{~Hz}$ & & & 1 & $0.99 \pm 0.00$ \\
\hline & LP $20 \mathrm{~Hz}$ & & & & 1 & LP $20 \mathrm{~Hz}$ & & & & 1 \\
\hline \multirow{4}{*}{ S5 } & & & & & & LP $0.5 \mathrm{~Hz}$ & 1 & $0.87 \pm 0.07$ & $0.97 \pm 0.02$ & $0.30 \pm 0.41$ \\
\hline & & & & & & LP $4 \mathrm{~Hz}$ & & 1 & $0.87 \pm 0.13$ & $0.75 \pm 0.25$ \\
\hline & & & & & & LP $10 \mathrm{~Hz}$ & & & 1 & $0.98 \pm 0.02$ \\
\hline & & & & & & LP $20 \mathrm{~Hz}$ & & & & 1 \\
\hline
\end{tabular}

DOTs \pm A MAD calculated for all possible comparisons of synergies extracted with different LP cut-off frequencies respectively in the 4 and 5 synergies case.

offs reduce the importance of the dominant muscles in explaining the variance of the original data. Low LP cut-offs increase the relative amplitude of the active muscles with respect to the inactive ones thus decreasing the signal-to-noise ratio of the weight coefficients. This is in agreement with the results previously reported by Shuman et al. (Shuman et al., 2017) and Krogt et al. (Krogt et al., 2016) for the lower-limb muscle synergies. Both studies recruited a cohort of healthy and cerebral-palsy children, and employed a walking task to test the influence of the preprocessing steps on the muscle synergies extracted from 5 task-relevant muscles. In line with our results, they have found, that also the Variance accounted for (VAF) criterion is sensitive to the LP cut-off frequency, with higher cut-offs resulting in lower VAF. Shuman et al. (Shuman et al., 2017) have also reported that the choice of the LP filter influences the lower-limb synergy weights coefficients and suggested that retaining the higher-frequency contented in the EMG signal, by using the higher LP cut-off, increases the synergy variability.

The reason behind very low ISS values for S5 for every LP cut-off frequency is a possible indication of noise affecting the synergy weights. In general, with high LP filter cut-offs (Table II) data tend to be noisier. On the other hand, very low cutoffs, such as $0.5 \mathrm{~Hz}$ are likely to oversmooth the signal, artificially raising the weights coefficients of the synergies and altering inter-subject similarity in an unpredictable way as a representative of the population. For this reason a careful assessment of individual differences in patients is necessary before employing ISS for quantification of the number of muscle synergies to extract. When muscle synergies are used e.g. (i) to reveal underlying patterns in muscle activity that may reflect different levels of neural functions in different motor tasks; (ii) as an assessment of neural deficits in muscle coordination for neurological subjects, ISS should not be used as a criterion to select the number of synergies, but rather as an extra informative feature. The ISS might however prove useful when muscle synergies are used for other purposes, such as to disentangle relevant information from noise, to simplify EMG analyses, to extract common activation patterns across individuals or to reduce the input features for a classifier.

\section{SELF normalization is equal to MVC normalization in terms synergy structure but it might have an impact on dimensionality}

Regarding normalization, there is no consensus as to its usefulness in accounting for variability (i.e., in physiological, anatomical and biochemical characteristics of the muscles). Both MVC and SELF normalization have been used in literature. For example, Coscia et al. used $\operatorname{MVC}[15,50,52]$, Clark et al. SELF normalization (maximum value across 
conditions) [24], Cheung et al. SELF normalization (median value across conditions or maximum variance) [21, 70]. MVC normalizes the amplitude of the EMG signal according to the maximum contraction that the muscle can yield, rather than to the maximum contraction achieved during the task performance as in SELF normalization. Without considering muscle synergies, normalization to MVC has been found by Halaki et al. to be the best one to compare muscle activity levels and activation patterns between muscles, tasks and individuals [71].

Our comparison reveals that the normalization method holds no effect on the weights of the muscle synergies. Yet, it definitely bears an effect on the cumulative variance criterion used to define the dimensionality of the data. In fact, while Eigenvalue $>1$ criterion was not affected by the normalization, the cumulative variance explained by a set of synergies was significantly lower with SELF than with MVC normalization. In fact, it may be unpractical to use MVC normalization for all muscles and especially with patients' populations. In this work we demonstrated that the type of normalization did not significantly alter the characteristics of the synergies extracted and we therefore show that SELF normalization can be safely employed for muscle synergies extraction

It is important to note however that SELF normalization gives equal importance to every muscle, regardless of their actual use in the movement. This might spread equally the variance also across unused muscles, thereby decreasing the variance explained by a set number of synergies. This should be taken into careful consideration when using muscle synergies dimensionality (i.e., number of extracted synergies according to a criterion) e.g. in rehabilitation, to determine the severity of movement impairment and/or to monitor the effect of a rehabilitative treatment [23].

In conclusion MVC and SELF normalization can be interchangeably chosen to normalize EMG signals for the muscle synergies extraction when the Eigenvalue $>1$ criterion is adopted to determine the number of the retained muscle synergies. However, our results suggest that particular care should be taken with other dimensionality selection criteria, especially when the dimensionality and the explained variance affect clinical interpretations of the study (e.g., effect of a post-stroke treatment)[24, 25, 70].

\section{Robustness of criteria to determine muscle synergies dimensionality}

We have found that the two different methods for determining muscle synergies dimensionality are differently affected by the normalization and smoothing (LP filtering) procedures. This is in agreement with the results obtained by Hug et al. [46] who showed that LP filter's cut-off frequency affects the number of synergies to be extracted (lower-limbs, cycling task) with different criteria.

In general, the number of synergies to retain is defined as the minimum number of muscle synergies able to capture the structural variation of the dataset so that, by adding one more synergy, only noise would be added to the reconstructed dataset [5]. Thresholds of $75 \%, 80 \%, 90 \%, 95 \%$ of explained variance have been adopted in the literature to find this particular number, according to the properties of the specific dataset. In particular, Coscia et al. [52] used 75\%, Cheung et al. [70] 80\%, d'Avella et al. [5] performed an iterative approach to determine the optimal number of synergies, resulting in an explained variance ranging from $73 \%$ to $82 \%$. In our case, we have chosen the explained variance threshold of $80 \%$.

The eigenvalue $\geq 1$ criterion did not show any dependence on the pre-processing steps (i.e., normalization, BP filtering and LP filtering). Indeed, the number of the synergies to be extracted was always equal to four. The explained variance criterion, instead, was sensitive to normalization type and LP filter. Regarding the LP filter, the higher the cut-off frequency the lower the cumulative variance and thus the higher the number of synergies to be extracted using this criterion. As the cut-off of the LP filter is lowered, the amount of smoothing applied to the data increases, which in turn reduces the complexity of the data and thus allows for higher cumulative variance explained by a set number of synergies. Though valuable in filtering out the noise, when used excessively, LP filter smoothing may facilitate information loss and thus influence the interpretation of the results. In general, algorithms less sensitive to pre-processing steps (e.g., eigenvalue criterion) should be used when possible. Recent clustering-based algorithms [37, 72] and the z-score normalized measure of synergy complexity suggested by Shuman et al. [48] may also help in increasing the robustness of the synergy extraction.

In some cases (with a homogeneous task and population) the measure of the inter-subject similarity may be used to finetune the number of synergies to extract, especially when the criteria used are not utterly conclusive. In our case, as it can be seen by Figures 3 and 4, the fifth extracted motor module, S5, could only capture a marginal portion of the EMG variance and may be thus considered not necessary to understand the main features of the global EMG data. However, the consistently higher index of the intra-subject similarity for the same muscles when using five motor synergies may indicate that using additional modules might prevent noise from spreading over the "good" synergies (S1, S2, S3, S4). In this particular case, it is possible to conclude that the set of five synergies is more robust than the set of four synergies. This result also shows that the "Eigenvalue $\geq 1$ " criterion, is robust to the pre-processing choices but may be too conservative in choosing the number of synergies to be extracted. A possibility to partially overcome this limitation is to modify the Eigenvalue threshold to $0.7 / 0.8$ as suggested by Joliffe et al. [73] or to increase by 1 the number of synergies yielded with the classical parameters.

\section{E. Limitations and future developments}

Comparisons were made by changing one pre-processing parameter at a time, which might lead to overlooking nonlinear interactions among pre-processing parameters. However, we believe non-linear effects to be negligible as the change of each parameter had a very specific and recognizable effect on the results. Further developments will include testing 
the robustness of these results in different tasks (e.g., walking, grasping), subject samples (e.g., elderly, post-stroke) and with different factorization algorithms (e.g., NMF, ICA).

\section{CONCLUSIONS}

Here we have determined for the first time how different pre-processing and amplitude normalization methods affect the synergies extracted with Factor Analysis in a 15-muscles upper-limb-reaching task. Results show that the preprocessing of the EMG signals is pivotal for muscle synergies analysis since all considered variables (normalization, filtering, and dimensionality reduction criterion) might influence the muscle synergies extraction. The choice of normalization method (SELF or MVC) does not alter the synergy structure but the SELF and MVC normalization are only equivalent if the Eigenvalue $>1$ criterion is adopted as a dimensionality reduction criterion. In fact, MVC normalization increases the explained variance by a set of synergies. Band-pass filtering ([20-500 Hz] or [50-500 Hz]) does not impact muscle synergies analysis but higher cut-offs increase inter-subject variability in some instances. The lowpass filtering cut-off frequency significantly alters the results: low cut-offs (e.g., $0.5 \mathrm{~Hz}$ ) increase the explained variance by a set of synergies and increase the contrast between active and inactive muscles but have an unpredictable effect on the intersubject similarity. We found that in some situations the intersubject similarity might be useful to complement common criteria in establishing the number of synergies to retain for further analyses. All considered it is advisable to carefully examine the pre-processing steps and in particular the LP cutoff frequencies, to appropriately address the requirements of both the experiment and the research questions, rather than solely relying on the values typically reported in the literature.

\section{ACKNOWLEDGEMENTS}

The authors would like to thank all the volunteers enrolled in the study. We thank Aurélie Sadaka-Stephan for the help with the recordings and Federica Servili for her preliminary (unpublished) work on this topic.

\section{REFERENCES}

[1] E. Bizzi, V. Cheung, A. d'Avella, P. Saltiel, and M. Tresch, "Combining modules for movement," Brain research reviews, vol. 57, no. 1, pp. 125-133, 2008.

[2] J. Roh, V. C. Cheung, and E. Bizzi, "Modules in the brain stem and spinal cord underlying motor behaviors," Journal of neurophysiology, vol. 106, no. 3, pp. 1363-1378, 2011.

[3] E. Burdet, D. W. Franklin, and T. E. Milner, Human robotics: neuromechanics and motor control. MIT press, 2013.

[4] N. A. Bernshteĭn. (1967). The co-ordination and regulation of movements.

[5] A. d'Avella, A. Portone, L. Fernandez, and F. Lacquaniti, "Control of fast-reaching movements by muscle synergy combinations," $J$ Neurosci, vol. 26, no. 30, pp. 7791-810, Jul 262006.

[6] A. d'Avella, L. Fernandez, A. Portone, and F. Lacquaniti, "Modulation of phasic and tonic muscle synergies with reaching direction and speed," J Neurophysiol, vol. 100, 2008.

[7] A. d'Avella, A. Portone, and F. Lacquaniti, "Superposition and modulation of muscle synergies for reaching in response to a change in target location," (in eng), $J$ Neurophysiol, Research Support, Non-U.S. Gov't vol. 106, no. 6, pp. 2796-812, Dec 2011.

[8] A. d'Avella, P. Saltiel, and E. Bizzi, "Combinations of muscle synergies in the construction of a natural motor behavior," (in eng), Nat Neurosci, Research Support, U.S. Gov't, P.H.S. vol. 6, no. 3, pp. 300-8, Mar 2003.

[9] G. Torres-Oviedo, J. M. Macpherson, and L. H. Ting, "Muscle synergy organization is robust across a variety of postural perturbations," J Neurophysiol, vol. 96, no. 3, pp. 1530-1546, 2006.

[10] S. A. Chvatal and L. H. Ting, "Common muscle synergies for balance and walking," Front Comput Neurosci, vol. 7, p. 48, 2013.

[11] Y. P. Ivanenko, R. E. Poppele, and F. Lacquaniti, "Distributed neural networks for controlling human locomotion: lessons from normal and SCI subjects," (in eng), Brain Res Bull, Review vol. 78, no. 1, pp. 13-21, Jan 152009.

[12] Y. P. Ivanenko et al., "Temporal components of the motor patterns expressed by the human spinal cord reflect foot kinematics," Journal of neurophysiology, vol. 90, no. 5, pp. 3555-3565, 2003.

[13] Y. P. Ivanenko, R. E. Poppele, and F. Lacquaniti, "Five basic muscle activation patterns account for muscle activity during human locomotion," The Journal of physiology, vol. 556, no. 1, pp. 267-282, 2004.

[14] S. A. Overduin, A. d'Avella, J. Roh, and E. Bizzi, "Modulation of muscle synergy recruitment in primate grasping," The Journal of Neuroscience, vol. 28, no. 4, pp. 880-892, 2008 .

[15] M. C. Tresch, P. Saltiel, and E. Bizzi, "The construction of movement by the spinal cord," Nat Neurosci, vol. 2, no. 2, pp. 162$167,1999$.

[16] F. A. Mussa-Ivaldi, S. F. Giszter, and E. Bizzi, "Linear combinations of primitives in vertebrate motor control," (in eng), Proc Natl Acad Sci US A, vol. 91, no. 16, pp. 7534-8, Aug 02 1994.

[17] M. A. Lemay and W. M. Grill, "Modularity of motor output evoked by intraspinal microstimulation in cats," (in eng), $J$ Neurophysiol, Comparative Study vol. 91, no. 1, pp. 502-14, Jan 2004.

[18] N. Dominici et al., "Locomotor primitives in newborn babies and their development," Science, vol. 334, no. 6058, pp. 997-9, Nov 18 2011.

[19] V. Monaco, A. Ghionzoli, and S. Micera, "Age-related modifications of muscle synergies and spinal cord activity during locomotion," J Neurophysiol, vol. 104, no. 4, pp. 2092-102, Oct 2010

[20] S. A. Safavynia, G. Torres-Oviedo, and L. H. Ting, "Muscle synergies: implications for clinical evaluation and rehabilitation of movement," Topics in spinal cord injury rehabilitation, vol. 17, no. 1, p. $16,2011$.

[21] V. C. Cheung, L. Piron, M. Agostini, S. Silvoni, A. Turolla, and E. Bizzi, "Stability of muscle synergies for voluntary actions after cortical stroke in humans," (in eng), Proc Natl Acad Sci U S A, Research Support, Non-U.S. Gov't vol. 106, no. 46, pp. 19563-8, Nov 172009

[22] J. Roh, W. Z. Rymer, E. J. Perreault, S. B. Yoo, and R. F. Beer, "Alterations in upper limb muscle synergy structure in chronic stroke survivors," (in eng), J Neurophysiol, vol. 109, no. 3, pp. 768-81, Feb 2013

[23] M. Coscia, V. Monaco, C. Martelloni, B. Rossi, C. Chisari, and S. Micera, "Muscle synergies and spinal maps are sensitive to the asymmetry induced by a unilateral stroke," (in eng), J Neuroeng Rehabil, Research Support, Non-U.S. Gov't vol. 12, p. 39, Apr 18 2015.

[24] D. J. Clark, L. H. Ting, F. E. Zajac, R. R. Neptune, and S. A. Kautz, "Merging of healthy motor modules predicts reduced locomotor performance and muscle coordination complexity poststroke," Journal of neurophysiology, vol. 103, no. 2, pp. 844-857, 2010.

[25] P. Tropea, V. Monaco, M. Coscia, F. Posteraro, and S. Micera, "Effects of early and intensive neuro-rehabilitative treatment on muscle synergies in acute post-stroke patients: a pilot study," Journal of neuroengineering and rehabilitation, vol. 10, no. 1, p. 103, 2013.

[26] E. Bizzi and V. C. Cheung, "The neural origin of muscle synergies," (in eng), Front Comput Neurosci, vol. 7, p. 51, 2013. 
F. Artoni et al., "Unidirectional brain to muscle connectivity reveals motor cortex control of leg muscles during stereotyped walking," Neuroimage, vol. 159, pp. 403-416, Aug 042017.

[38] F. Artoni, E. Pirondini, A. Panarese, and S. Micera, "Exploring Neuro-Muscular Synergies of Reaching Movements with Unified Independent Component Analysis," IEEE EMBC 2016, 2016.

[39] F. Artoni, A. Gemignani, L. Sebastiani, R. Bedini, A. Landi, and D. Menicucci, "ErpICASSO: a tool for reliability estimates of independent components in EEG event-related analysis," Conf Proc IEEE Eng Med Biol Soc, vol. 2012, pp. 368-71, 2012.

[40] L. A. Merkle, C. S. Layne, J. J. Bloomberg, and J. J. Zhang, "Using factor analysis to identify neuromuscular synergies during treadmill walking," J Neurosci Methods, vol. 82, no. 2, pp. 207214, 1998

[41] G. Torres-Oviedo and L. H. Ting, "Muscle synergies characterizing human postural responses," J Neurophysiol, vol. 98, no. 4, pp. 2144-2156, 2007

[42] B. L. Davis and C. L. Vaughan, "Phasic behavior of EMG signals during gait: use of multivariate statistics," Journal of Electromyography and Kinesiology, vol. 3, no. 1, pp. 51-60, 1993.

[43] A. M. Sabatini, "Identification of neuromuscular synergies in natural upper-arm movements," Biological cybernetics, vol. 86, no. 4, pp. 253-262, 2002.

[44] F. Artoni, V. Monaco, and S. Micera, "Selecting the best number of synergies in gait: preliminary results on young and elderly people," IEEE Int Conf Rehabil Robot, vol. 2013, p. 6650416, Jun 2013.

[45] N. Lambert-Shirzad and H. F. Van der Loos, "On identifying kinematic and muscle synergies: a comparison of matrix factorization methods using experimental data from the healthy population," (in eng), J Neurophysiol, vol. 117, no. 1, pp. 290-302, Jan 012017

[46] F. Hug, N. A. Turpin, S. Dorel, and A. Guével, "Smoothing of electromyographic signals can influence the number of extracted muscle synergies," Clinical Neurophysiology, vol. 123, no. 9, pp. 1895-1896, 2012.

[47] M. Krogt, L. Oudenhoven, A. Buizer, A. Dallmeijer, N. Dominici, and J. Harlaar, "The effect of EMG processing choices on muscle synergies before and after BoNT-A treatment in cerebral palsy," Gait \& Posture, vol. 49, p. 31, 2016/09/01/2016.
[48] B. R. Shuman, M. H. Schwartz, and K. M. Steele, "Electromyography Data Processing Impacts Muscle Synergies during Gait for Unimpaired Children and Children with Cerebral Palsy," Frontiers in Computational Neuroscience, vol. 11, p. 50, 2017

[49] E. Pirondini, M. Coscia, A. Crema, M. Mancuso, and S. Micera, "How the selection of muscles influences their synergies? A preliminary study using real data," in Neural Engineering (NER), 2013 6th International IEEE/EMBS Conference on, 2013, pp. 581584: Ieee.

[50] E. Pirondini et al., "Evaluation of the effects of the Arm Light Exoskeleton on movement execution and muscle activities: a pilot study on healthy subjects," Journal of NeuroEngineering and Rehabilitation, journal article vol. 13, no. 1, pp. 1-21, 2016.

[51] A. Sadaka-Stephan, E. Pirondini, M. Coscia, and S. Micera, "Influence of trajectory and speed profile on muscle organization during robot-aided training," in Rehabilitation Robotics (ICORR), 2015 IEEE International Conference on, 2015, pp. 241-246: IEEE.

[52] M. Coscia, V. C. Cheung, P. Tropea, A. Koenig, V. Monaco, and C. Bennis, "The effect of arm weight support on upper limb muscle synergies during reaching movements," J Neuroeng Rehab, vol. $11,2014$.

[53] H. J. Hermens, B. Freriks, C. Disselhorst-Klug, and G. Rau, "Development of recommendations for SEMG sensors and sensor placement procedures," Journal of electromyography and Kinesiology, vol. 10, no. 5, pp. 361-374, 2000.

[54] H. M. Clarkson, Musculoskeletal assessment: joint range of motion and manual muscle strength. Lippincott Williams \& Wilkins, 2000.

[55] L. Daniels and C. Worthingbam, "Muscle Testing, Techniques of Manual Examination," American Journal of Physical Medicine \& Rehabilitation, vol. 53, no. 5, p. 241, 1974

[56] A. d'Avella and M. Tresch, Muscle Synergies for Motor Control.

[57] A. Ajiboye and R. Weir, "Muscle synergies as a predictive framework for the EMG patterns of new hand postures," Journal of neural engineering, vol. 6, no. 3, p. 036004, 2009.

[58] L. J. Myers et al., "Rectification and non-linear pre-processing of EMG signals for cortico-muscular analysis," (in eng), J Neurosci Methods, Research Support, Non-U.S. Gov't vol. 124, no. 2, pp. 157-65, Apr 152003

[59] Y. P. Ivanenko, G. Cappellini, N. Dominici, R. E. Poppele, and F. Lacquaniti, "Coordination of locomotion with voluntary movements in humans," Journal of Neuroscience, vol. 25, no. 31, pp. 7238-7253, 2005.

[60] Y. P. Ivanenko, R. E. Poppele, and F. Lacquaniti, "Motor control programs and walking," The Neuroscientist, vol. 12, no. 4, pp. 339348, 2006.

[61] J. M. Wakeling and T. Horn, "Neuromechanics of muscle synergies during cycling," Journal of neurophysiology, vol. 101, no. 2 , pp. $843-854,2009$.

[62] A. Danna-dos-Santos, K. Slomka, V. M. Zatsiorsky, and M. L. Latash, "Muscle modes and synergies during voluntary body sway," Experimental Brain Research, vol. 179, no. 4, pp. 533-550, 2007.

[63] T. Asaka, Y. Wang, J. Fukushima, and M. L. Latash, "Learning effects on muscle modes and multi-mode postural synergies," Experimental Brain Research, vol. 184, no. 3, pp. 323-338, 2008.

[64] V. Krishnamoorthy, M. L. Latash, J. P. Scholz, and V. M. Zatsiorsky, "Muscle synergies during shifts of the center of pressure by standing persons," Experimental brain research, vol. 152, no. 3, pp. 281-292, 2003.

[65] Gizzi L, Nielsen JF, Felici F, Y. P. Ivanenko, and D. Farina, "Impulses of activation but not motor modules are preserved in the locomotion of subacute stroke patients," J Neurophysiol, vol. 106, pp. 202-210, 2011.

[66] D. Torricelli et al., "Muscle synergies in clinical practice: theoretical and practical implications," in Emerging Therapies in Neurorehabilitation II: Springer, 2016, pp. 251-272.

[67] K. M. Steele, A. Rozumalski, and M. H. Schwartz, "Muscle synergies and complexity of neuromuscular control during gait in cerebral palsy," Developmental Medicine \& Child Neurology, vol. 57 , no. 12, pp. 1176-1182, 2015.

[68] F. O. Barroso et al., "Shared muscle synergies in human walking and cycling," Journal of neurophysiology, vol. 112, no. 8, pp. 1984-1998, 2014 
[69] R. L. Routson, D. J. Clark, M. G. Bowden, S. A. Kautz, and R. R. Neptune, "The influence of locomotor rehabilitation on module quality and post-stroke hemiparetic walking performance," Gait \& posture, vol. 38, no. 3, pp. 511-517, 2013.

[70] V. C. Cheung et al., "Muscle synergy patterns as physiological markers of motor cortical damage," Proc Natl Acad Sci USA, vol. 109 , pp. 14652-14656, 2012.

[71] M. Halaki and K. Ginn, "Normalization of EMG Signals: To Normalize or Not to Normalize and What to Normalize to?," Current applications and future challenges, p. 175, 2012.
[72] Y. Kim, T. C. Bulea, and D. L. Damiano, "Novel methods to enhance precision and reliability in muscle synergy identification during walking," Frontiers in human neuroscience, vol. 10, 2016.

[73] I. T. Jolliffe, "Discarding Variables in a Principal Component Analysis. I: Artificial Data," Journal of the Royal Statistical Society. Series C (Applied Statistics), vol. 21, no. 2, pp. 160-173, 1972. 